CPR decision was completed in 100\%, with 94\% DNACPR (range $86-100 \%$ ). A capacity decision was recorded in $93 \%$ and emergency contacts in 38\% (range 8-88\%).

Conclusions The process has been embedded across Coventry and Warwickshire, however more care home residents could be supported and old DNACPR paperwork should be reviewed. Nurse led completion is more evident in the community. Documentation of patient involvement using the optional personal preference section is limited. The form is widely used as a DNACPR form; the potential for documenting the goal of care and specific clinical recommendations is not being fully utilised and should be a focus for training. Patients and carers could be encouraged to complete the emergency contacts section.

\section{1 SPECIALTY 3 SETTINGS: THE INFLUENCE OF COMBINED ONCOLOGY AND PALLIATIVE CARE CLINICS (COPC) ON CONTACTS WITH PALLIATIVE CARE IN HOSPITAL, COMMUNITY AND HOSPICE IN CORNWALL}

Naomi Blower, Rhys Elgumati, Rachel Newman, Natasha Powell, Kirsty Scott, Ziad Zeidan. University of Exeter Medical School, Cornwall Hospice Care, Royal Cornwall Hospitals NHS Trust

\subsection{6/spcare-2020-PCC.191}

Background Evidence suggests palliative care involvement alongside oncology care improves quality of life, planning and communication for advanced cancer patients. However, little information exists about the impact of such involvement on access to the speciality across care settings. This study examines the influence of COPC versus Standard Oncology Clinics (SOC) on palliative care contacts in hospital, community and hospice settings.

Methods Fifty-nine COPC patients were compared with fiftynine SOC clinic patients, all with lung malignancies. 56/59 COPC patients and 59/59 SOC patients were first seen within 2017-2018. Data about involvement of community and hospice palliative care services was collected for 12 months from first contact with palliative medicine oncology consultant respectively. Hospital palliative care involvement was measured from 1 month before to 12 months after first consultant contact (thereby including those first diagnosed during a hospital admission).

Results Hospital palliative care services provided 28 care episodes in 20/59 (34\%) COPC patients, compared to 19 in $14 /$ 59 (24\%) SOC patients. Patients were already known to community palliative care teams in $15 / 59$ patients attending COPC increasing to $46 / 59$ within next 12 months. In SO the figures were $8 / 59$ increasing to $24 / 59$. Although higher numbers in the COPC group, the rate of increase is similar (approximately $300 \%$ increase over 12 months in each group) $7 / 59$ (11\%) of COPC compared with $3 / 59(5 \%)$ of SO patients were admitted to a local hospice in 12 months from first contact.

Conclusions Review in COPC compared to SOC appears to increase access to hospital, community and hospice specialist palliative care services. This study involves a relatively small number of patients with lung malignancies, and larger studies, including patients with other types of malignancy, should expand the information available. Further data is also needed to evidence whether access to these services improves outcomes for patients and families.

\section{THE INFLUENCE OF COMBINED ONCOLOGY AND PALLIATIVE CARE CLINICS (COPC) ON HOSPITAL ADMISSIONS AND VARIOUS OUTCOMES FOR PATIENTS WITH LUNG MALIGNANCIES IN CORNWALL}

Naomi Blower, Rhys Elgumati, Rachel Newman, Natasha Powell, Kirsty Scott, Ziad Zeidan. University of Exeter Medical School, Cornwall Hospice Care, Royal Cornwall Hospitals NHS Trust

\subsection{6/spcare-2020-PCC.192}

Background Evidence suggests early palliative care involvement (PCI) alongside oncology care improves quality of life, planning and communication for advanced cancer patients. Previous work in Cornwall suggested COPC reduced number and length of hospital admissions in oncology patients with varied cancer diagnoses. This study examines findings in lung cancer patients.

Methods Baseline data was collected for fifty-nine patients attending lung cancer COPC or Standard Oncology Clinic (SOC), including (but not limited to) mortality rates, average survival of those who died, performance status, and stage of disease. Over 12 months from initial oncology or palliative medicine consultant contact, rates of hospital admissions, length of stay, reasons for admission, and preferred place of discharge were recorded.

Results Background data suggested there was little difference in the baseline data for patients attending the 2 clinic types, except that performance status was somewhat worse for the COPC group (ECOG for COPC patients $1=19,4=8$, versus SOC $1=25,4=2$ with ECOG 2 and 3 same number each group). Mortality rates were similar. Of deaths, survival was 4.82 months COPC versus 5.33 SOC. COPC patients had 56 hospital admissions, and SOC 49. Average Length of stay was similar across the 2 groups. Reason for admission differed between the 2 groups: COPC/SOC complication of disease $(60.7 \%$ versus $44.9 \%)$ COPC/SOC complication of treatment $(12.5 \%$ versus $38.8 \%)$. COPC recorded Preferred Place of Discharge in $37.3 \%$ of admissions versus SOC $18.6 \%$. Rates of achievement were similar. COPC recorded other advance care planning in $41.0 \%$ patients versus $10.2 \%$ SOC.

Conclusions In lung cancer patients rates of hospital admissions and LOS were not reduced by COPC input, but reasons for admission were different. Despite poorer performance status results there was no difference in the average number of months survival of those who died within the 12 months analysed.

\section{NON-INVASIVE TECHNOLOGY TO SUPPORT CLINICAL CARE IN PALLIATIVE HEAD AND NECK CANCER: A SYSTEMATIC REVIEW OF THE USE OF BIOELECTRICAL IMPEDANCE ANALYSIS FOR BODY COMPOSITION ASSESSMENTS}

Amara C Nwosu, Catriona R Mayland. Palliative Care Institute Liverpool, University of Liverpool, Sheffield Teaching Hospitals NHS Foundation Trust

\subsection{6/spcare-2020-PCC.193}

Background/Aims Head and neck cancer is associated with significant pathophysiological impacts on body composition, which can create problems such as dehydration, poor nutrition and cachexia. Bioelectrical impedance analysis is a non-invasive body composition assessment tool which is increasingly being 
used to undertake nutritional assessment and measure clinical outcomes, such as prognosis. To date, no studies have reviewed the use of bioelectrical impedance analysis in head and neck cancer. This study aims to synthesise the different applications of bioelectrical impedance analysis in the clinical assessment of adults affected with advanced head and neck cancer.

Methods A systematic review of literature using five electronic databases (PubMed, Medline, EMBASE, CINAHL and PsychINFO). Search terms were based on 'head and neck cancer' and various terms relating to 'bioelectrical impedance analysis'. Empirical studies reporting the use of the different forms of bioelectrical impedance analysis in advanced head and neck cancer were included.

Results The database search identified 31 articles, of which 17 were included for full review. Bioelectical impedance analysis has been used for several purposes with patients with head and neck cancer, such as prognostication and assessments of hydration, nutrition, mucositis and fatigue. Only three articles focused specifically on the palliative phase of illness, and just one article focused on the clinical application in palliative care (predicting prognosis).

Conclusion Bioelectrical impedance analysis shows promise as a tool to assess body composition and help direct clinical care in head and neck cancer. Little evidence exists, however, for its use within palliative care and this represents an area for future focus to help with clinical decision-making and wider clinical application.

\section{EVALUATING AN 8 WEEK MINDFULNESS PROGRAMME DEVELOPED TO SUPPORT HOSPICE STAFF AND VOLUNTEERS AT LOROS HOSPICE}

Barbara Powell, Angela Baxter. LOROS Hospice

\subsection{6/spcare-2020-PCC.194}

Background Literature recognises the benefits of mindfulness practice on resilience, self-care and emotional intelligence. Front line hospice staff are at risk of compassion fatigue and burnout. An opportunity allowed us to assess the impact of an 8 week course on the well-being and self-compassion of hospice staff and volunteers.

Method A mindfulness based cognitive therapy (MBCT) course was developed encouraging participants to re-evaluate their responses to challenging situations and promoted self-care and compassion. Courses were open to all interested hospice staff and volunteers. Six courses were delivered over a ten month period. Participants completed pre and post-course WarwickEdinburgh Mental Well-being Scale (WEMWBS) questionnaires and provided free text reflection.

Results Courses involved 80 participants and four facilitators. Groups were 'closed' for the duration of the course; participants had a wide variety of roles within the hospice. WEMWBS maximum possible score $=70, \quad$ minimum $=14$; National average $=53$ (Health England Survey 2011)

53 completed pre and post questionnaires were matched and analysed.

Average pre-course score $=45.1$ (range 20-63), average postcourse score $=54.5$ (range 35-68)

Average change $=+9.3$.

$87 \%$ rated the course as of significant benefit.

98\% planned to maintain a mindfulness practice.
Free text feedback was considerable and included:

'I feel much calmer, supportive of others and it has stopped me from over-thinking situations that I can't change'

'Using different tool to adapt to the time you have'

'Being aware of thoughts and refocusing'

'Thank you for showing me I am important too and that only I can change things'

Conclusions Both WEMWBS scores and free text feedback suggest significant perceived benefit to participants. Feeling valued and more resourceful when faced with challenging situations are common themes. Long term benefits to participants and impact on patient care are still to be measured.

\section{SUBSTANCE MISUSE IN PALLIATIVE CARE: HOW WELL DO WE DOCUMENT KEY INFORMATION FOR EFFECTIVE MULTI-AGENCY WORKING ACROSS HEALTHCARE SETTINGS IN MERSEY?}

Penny Shepherd, Joanna Roberts, Kamilla Krueger, Sabine Auth, Elaine Mannering, Malcolm Cooper, Laura Chapman, Jenny Smith, Anthony Thompson. Woodlands Hospice, Willowbrook Hospice, Merseycare, Clatterbridge Cancer Centre, Marie Curie Liverpool, Countess of Chester Hospital, St Helens and Knowsley Teaching Hospitals

\subsection{6/spcare-2020-PCC.195}

Background Substance misuse (including alcohol) is a growing issue in the UK and can have far reaching effects on wellbeing, symptoms, compliance, social support and ability to access services. Medications with a potential for misuse are used commonly in palliative care. Patients with substance misuse should receive the same standard of care and treatment of symptoms as other patients. Providing this care across settings and reducing risks requires multi-agency working and good communication between healthcare professionals. Regional guidance relating to this exists in Mersey from 2009.

Aims To review current documentation of key information in patients known to palliative care with substance misuse in the Mersey region as part of updating the regional guidance.

Methods A retrospective multi-centre case-note audit was conducted including documentation of key information in patients with life limiting illness and known substance misuse receiving specialist palliative care in hospital, hospice and community settings.

Results 73 cases across the three settings were included (22\% community, 40\% Hospice, 37\% Hospital). $73 \%$ had documentation of whether drug and alcohol services were involved, $53 \%$ had documentation of whether a key worker was involved. For the 24 patients on opioid-substitution therapy (OST) 22\% had information about their community pharmacist documented, $74 \%$ had documentation of their named prescriber for OST and 63\% had documentation of their named prescriber for opioids. Documentation of communication with community teams following changes to opioid medications varied: GPs $61 \%$, drug and alcohol teams $12 \%$, and community pharmacists $5 \%$. The method of communication also varied.

Conclusions This audit highlighted inconsistent documentation of important information relating to substance misuse in patients with life limiting illness that could impair communication between healthcare professionals and services, potentially affecting the care and safety of these patients. These results have helped to inform the update of the regional guidelines in Mersey. 